\title{
EL IMPACTO DE LA RECESIÓN ECONÓMICA SOBRE LA SITUACIÓN Y LA DISCRIMINACIÓN DE LA MUJER INMIGRANTE TRABAJADORA EN ESPAÑA. ESTUDIO DE CASO DE LA REGIÓN DEL PAÍS VASCO
}

\author{
The impact of economic crisis on the situation and discrimination \\ of the immigrant working women in Spain. The case of the \\ Basque Country
}

\author{
María José Martín Herrero ${ }^{7}$ \\ Maite Fouassier Zamalloa? \\ Gorka Moreno Márquez
}

\begin{abstract}
Resumen. Este artículo tiene por objetivo dibujar la situación de las mujeres inmigrantes en la Comunidad Autónoma del País Vasco (CAPV) en el ámbito laboral, tomando como referencia dos periodos temporales distintos, en 2010 cuando la crisis económica global comienza a acechar a la economía vasca, y en 2014 una vez avanzada y que permite valorar su impacto en este colectivo. Tal comparación nos permitirá analizar la evolución en el mercado laboral y la respuesta de estas mujeres, generalmente protagonistas de situaciones de mayor vulnerabilidad y discriminación.
\end{abstract}

Palabras clave: mujeres inmigrantes, discriminación, mercado laboral, País Vasco, crisis.

\begin{abstract}
This article aims to draw the situation of immigrant women in the labor market in the Basque Country. We study two different periods, 2010 when the global crisis appears in the basque economy, and 2014, when we can value the impact on this group. This comparison will allow us to analyze the evolution in the labor market and the response of these women, who have experienced situations of greater vulnerability and discrimination.
\end{abstract}

Keywords: Immigrant women, discrimination, labour market, Basque Country, crisis.

\footnotetext{
1 Ikuspegi - Observatorio Vasco de Inmigración. Universidad del País Vasco. Leioa, Bizkaia, España.
} 


\section{Introducción}

La crisis económica global de la que poco a poco nos estamos recuperando según algunos analistas, ha resultado especialmente dura con el colectivo inmigrante, que desde sus inicios comenzó a sufrir las consecuencias de una recesión que afectó, entre otras y especialmente, al mundo laboral. Si bien a finales de los noventa del siglo pasado se produjo en España un crecimiento destacado de llegadas de población extranjera, destinada a cubrir las necesidades del mercado de trabajo, es a partir de 2008, y debido al inicio de la crisis, cuando se perciben algunos indicadores que sitúan a la población extranjera como uno de los colectivos más castigados. Así lo corroboran algunos datos referentes a la ocupación y paro en España, que sitúan a la población extranjera en tasas muy superiores de desempleo en comparación con la población autóctona y con algunos indicadores que delatan cierta precarización laboral y salarial, afectando al resto de las esferas de la vida. Del mismo modo que la actividad económica y la riqueza del Estado decrecen, los flujos migratorios también viven el cambio, con menos recepción de población extranjera -que no su ausenciaacomodándose, en definitiva, a la realidad socio-económica del momento. En el caso de la Comunidad Autónoma del País Vasco (CAPV en adelante), la crisis también es una realidad, aunque su aparición fue algo más tardía que en el resto del Estado, y con características diferenciadas debido a la estructura del mercado laboral, con el sector servicios como el principal motor de la economía vasca. Su impacto afectó a la población extranjera en términos de destrucción de puestos laborales en donde estaba empleada, un aumento del desempleo y variaciones en los sectores de actividad en los que está trabajando, entre otros. Actualmente se observan nuevos datos que indican un aumento en los movimientos migratorios, unos números a los que deberemos prestar atención de ahora en adelante, coincidiendo con la leve mejoría de la economía.

El modelo migratorio vasco aparece como ejemplo paradigmático de los nuevos flujos, que rompen con el esquema de análisis clásico de las migraciones, y del peso en los mismos de las mujeres inmigrantes, que pasan a ser un agente activo y protagonista en el proyecto migratorio, sobre todo unido a las necesidades en el mercado laboral del país de destino para hacer frente a las necesidades sociales y demográficas de la población autóctona² ${ }^{2}$

Este artículo pretende prestar atención concretamente a las mujeres extranjeras, ya que entendemos que dentro del colectivo inmigrante son las que presentan mayor vulnerabilidad y discriminación, en este caso, en el ámbito laboral. Para ello analizamos algunos indicadores de actividad laboral a partir de la Encuesta de Población Inmigrante Extranjera (EPIE en adelante), una operación estadística oficial cuatrienal realizada por el Departamento de Empleo y Políticas Sociales del Gobierno Vasco, en dos periodos diferentes: el primero en el año

\footnotetext{
2 MORENO, Gorka. El impacto de la crisis sobre el colectivo inmigrante en el País Vasco.
} 
2010 cuando la crisis se hace evidente en la CAPV, y en el año 2014, cuando el impacto de la crisis puede ser ya estudiado y valorado, si bien algunos indicadores económicos señalan esta fecha como el comienzo de cierta recuperación.

La primera parte del artículo recoge el estado de la cuestión del mercado de trabajo y su importancia como elemento fundamental ante la llegada de población extranjera. En concreto, nos detenemos a analizar el papel de las mujeres inmigrantes como iniciadoras del proyecto migratorio, sea familiar o individual, en busca de mejores condiciones de vida, y a describir algunas características y factores a destacar de su inserción laboral en ciertos sectores de actividad. Posteriormente presentamos la metodología utilizada en este artículo y algunos resultados basados en el análisis de las tasas de actividad, ocupación y paro de la población femenina de origen extranjero residente en la CAPV, según área geográfica de origen, y los sectores de actividad en los que se insertan, siempre comparando los datos referentes a 2010 y 2014. Por último, se presentan algunas conclusiones que nos resultan interesantes de subrayar.

\section{Las mujeres inmigrantes en el mercado laboral español}

El papel y la relevancia del empleo y el mercado laboral en los proyectos migratorios y en los procesos de integración del colectivo inmigrante son un tema recurrente dentro del estudio de las migraciones internacionales, tanto a nivel internacional ${ }^{3}$, como para el caso de España ${ }^{4}$, convirtiéndose así en muchos casos en el eje que vertebra todo el proceso migratorio, la integración en el país receptor y también los efectos del propio proyecto en origen.

En estas últimas décadas se ha hecho hincapié en el rol y el impacto del empleo sobre las mujeres migrantes, un tema que anteriormente había sido relegado a un segundo plano y que de la mano del aumento de los flujos migratorios femeninos ha tomado fuerza dentro del análisis de los movimiento migratorios ${ }^{5}$. De hecho, en la actualidad casi la mitad de las personas migrantes en el mundo son mujeres ${ }^{6}$ y este porcentaje se sitúa en el caso de Europa incluso por encima del $50 \%$.

Todo ello en un escenario en el que las mujeres inmigrantes dejan de ser la acompañante de los hombres en el proyecto migratorio y pasan a ser en muchas ocasiones las protagonistas del mismo, con unas marcadas motivaciones económicas y laborales en un contexto de globalización económica ${ }^{8}$.

3 PIORE, Michael. Birds of passage: migrant labor and industrial societies; CASTLES, Stephen, MILLER, Mark J. The Age of Migration.

4 CACHÓN, Lorenzo. Integración de los inmigrantes a través del trabajo; CARRASCO, Concepción. Inmigración y mercado de trabajo. Informe 2011.

5 KOFMAN, Eleonore. Gendered Global Migrations; PIPER, Nicola. Gender and Migration.

6 ZLOTNIK, Hania. The Global Dimensions of Female Migration; UNITED NATIONS. International Migration Report 2013.

7 MORRISON, Andrew R., SCHIFF, Maurice, SJÖBLOM, Mirja. The international migration of women.

8 GREGORIO, Carmen. La migración femenina y su impacto en las relaciones de género; OROZCO, Amaia. Cadenas Globales de Cuidados. 
Esta inserción en los mercados laborales globalizados, se da, en gran parte, dentro de una marcada segmentación en la que el conjunto de la población inmigrante, y más en concreto las mujeres, ocupa los puestos de trabajo más precarios, informales y de baja cualificación ${ }^{9}$. En el caso concreto de las mujeres el sector de las tareas domésticas y los cuidados personales es fundamental y es uno de los que explican en gran parte este proceso anteriormente citado de feminización de las migraciones económicas a nivel mundial ${ }^{10}$.

El modelo inmigratorio español no es ajeno a estas dinámicas y a partir del año 2000 la llegada de población extranjera ha sido muy intensa, por lo menos hasta la irrupción de la crisis económica, a partir de 2007-2008. De hecho, en el periodo que va de 2000 a 2008 España ha sido el país de la OCDE que más población extranjera ha recibido en términos relativos y el segundo en términos absolutos, tan sólo por detrás de Estados Unidos ${ }^{11}$. Esta inmigración, en gran parte inesperada ${ }^{12}$, ha supuesto un importante cambio demográfico y un aumento de la población residente en España, que ha pasado de 39.852.651 personas en 1998 a 46.524 .943 en 2016. Del mismo modo, y en este mismo periodo, la población nacida en el extranjero ha pasado, según el Instituto Nacional de Estadística español, de 1.173.767 personas a 6.109.272.

El modelo inmigratorio español se ha situado en un contexto de crecimiento económico y de demanda de mano de obra intensiva y temporal, generalmente de baja cualificación y que ha ocupado los puestos de trabajo más precarios dentro de la escala laboral española ${ }^{13}$, junto a estas características también hay que destacar la escasa posibilidad de movilidad laboral ascendente ${ }^{14}$.

En el caso de las mujeres esta pauta se han dado con más intensidad aún si cabe ${ }^{15}$, debido a las características propias de los sectores laborales en los que participan principalmente. Sectores, como la hostelería y el comercio y sobre todo el de las tareas domésticas y de los cuidados, que sufren una desregulación de las condiciones laborales todavía mayor que otros sectores donde el peso de

9 PARELLA, Sonia. Las lógicas de la discriminación de las mujeres inmigrantes en el mercado de trabajo. Mecanismos y repercusiones.

${ }^{10}$ GLENN, Evelyn N. From Servitude to Service Work: The Historical Continuities of Woemen's Paid and Unpaid Reproductive Works; SASSEN, Saskia. Why Migration? Tesis contra los modelos de explicación al uso; HOSCHSCHILD, Arlie R. Las cadenas mundiales de afecto y asistencia y la plusvalía emocional.

${ }^{11}$ OECD. International Migration Outlook.

12 IZQUIERDO, Antonio. La inmigración inesperada: La población extranjera en España (1991-1995).

${ }^{13}$ COLECTIVO IOÉ. Mujeres inmigrantes en España. Proyectos migratorios y trayectorias de género; CACHÓN, Lorenzo. Diez notas sobre la inmigración en España y una conclusión ni inesperado ni desesperada; CACHÓN, Lorenzo. La España inmigrante: marco discriminatorio, mercado de trabajo y políticas de integración; PAJARES, Miguel. Inmigración y mercado de trabajo. Informe 2010.

${ }^{14}$ CEBOLLA, Héctor, GONZÁLEZ-FERRER, Amparo. La inmigración en España (2000-2007). De la gestión de los flujos a la integración de los inmigrantes.

${ }^{15}$ OSO, Laura. La migración hacia España de mujeres jefes del hogar; COLECTIVO IOÉ. Mujer, inmigración y trabajo. 
los hombres inmigrantes ha podido ser mayor, como la construcción o el sector primario.

De esta manera, el sector de las tareas domésticas y los cuidados personales se ha erigido en España como uno de los principales nichos laborales para las mujeres inmigrantes ${ }^{16}$, más aún en aquellas regiones en las que el peso del turismo o el sector primario no son importantes -Madrid y zona norte-, aunque tiene relevancia en todo el conjunto nacional.

El aumento del acceso al mercado laboral de las mujeres autóctonas junto con el envejecimiento de la población provocó la necesidad de mano de obra que se hiciera cargo de las tareas domésticas y del cuidado. De ahí la demanda de población femenina inmigrante para hacer frente a tales carencias ${ }^{17}$, sobre todo en el caso de las mujeres de origen latinoamericano ${ }^{18}$.

Este sector se ha convertido en gran parte de los casos en la puerta de acceso al mercado laboral para la mujer inmigrante. Según se avanzaba en el proyecto y en estabilidad administrativa y social normalmente ha transitado hacia otros sectores como la hostelería o el comercio $^{19}$.

Tal evolución se ha visto modificada, en parte, con la irrupción de la recesión económica a partir del año 2008 y los años posteriores hasta llegar a 2014. A partir de esta fecha, la actividad económica española, medida a partir de su Producto Interior Bruto (PIB) experimentó un crecimiento interanual positivo y mayor a la media de la $\cup E^{20}$. De hecho, en algunos casos, sobre todo entre la población latinoamericana femenina, se ha podido observar cómo si en periodo de bonanza económica se transitaba desde el sector de las tareas domésticas hacia otros con mejores condiciones laborales ${ }^{21}$, en el periodo de crisis este mismo sector se ha convertido en un nicho laboral refugio, detectando en algunos casos trayectorias inversas de vuelta a las tareas domésticas. Es más, dicho retorno, incluso, se ha convertido en una estrategia de respuesta ante la crisis para abaratar gastos, como por ejemplo en el caso de aquellas mujeres

${ }_{16}$ DIAZ GORFINKIEL, Magdalena. El mercado de trabajo de los cuidados y la creación de las cadenas globales de cuidado: ¿cómo concilian las cuidadoras?; MOLPECERES, Laura. Situación laboral de las mujeres inmigrantes en España; OIM. Estudio sobre la situación laboral de la mujer inmigrante en España.

17 TORNS, Teresa. Los servicios de proximidad zun yacimiento de empleo?; PARELLA, Sonia. El trasvase de desigualdades de clase y etnia entre mujeres: los servicios de proximidad; GARCÍA, Cristina et alii. La construcción social del mercado laboral doméstico en España a comienzos del siglo XXI.

${ }^{18}$ GIL, Sandra, GONZALEZ-FERNÁNDEZ, Tania. International migration, public policies and domestic work: Latin American migrant women in the Spanish domestic work system.

${ }^{19}$ COLECTIVO IOÉ. Mujer..., op. cit.

${ }^{20}$ CONSEJO ECONÓMICO Y SOCIAL. Memoria sobre la situación socioeconómica y laboral de España 2015.

${ }^{21}$ MARTíNEZ BUJÁN, Raquel. iEl trabajo doméstico cuenta! Características y transformaciones del servicio doméstico en España. 
que han optado por convertirse en trabajadoras domésticas internas, para así ahorrar el costo derivado de la vivienda o la manutención ${ }^{22}$.

Así, la crisis económica que ha sufrido España ha marcado un antes y un después en el desarrollo del modelo inmigratorio. La llegada masiva de población extranjera se ha ralentizado de manera muy marcada en este periodo e incluso ha descendido en algunas regiones, aunque los fenómenos de retorno no han sido para nada masivos ${ }^{23}$.

Se ha notado especialmente el impacto de la crisis sobre el colectivo inmigrante en el ámbito laboral ${ }^{24}$. En un periodo relativamente corto de tiempo -de 2007 a 2010- se destruyen 2,1 millones de puestos de trabajo de personas inmigrantes, de ellos 1,1 en el sector de la construcción, que es eminentemente masculino ${ }^{25}$. De esta manera, el desempleo de la población extranjera en España pasó del 11,8\% en el tercer trimestre de 2007 al 39,2\% en el primero de 2013. Mientras que la ocupación ha descendido para el colectivo inmigrante en un 25,2\%, para la autóctona lo ha hecho en un $12,4 \%{ }^{26}$.

El impacto de la crisis no sólo ha tenido efecto en términos de ocupación y desempleo, sino que ha hecho que las ya de por sí precarias condiciones laborales del colectivo inmigrante se hayan deteriorado todavía más ${ }^{27}$.

Estos datos inciden en una pauta que se ha dado a lo largo de la crisis, la población inmigrante ha sufrido el envite de la crisis en el mercado laboral con mayor intensidad que la autóctona. Este rasgo, que también se da en el plano internaciona ${ }^{28}$, en el caso de España se ha dado sobre todo en el primer periodo de la crisis - de 2007 a 2010-29, mientras que parece que en el segundo periodo de la crisis - de 2011 a 2014- la distancia entre ambos colectivos no ha aumentado tanto.

En el caso específico del colectivo inmigrante la crisis también ha influido de diferente manera según el sexo. En el periodo previo a la crisis la tasa de ocupación masculina era mayor que la femenina, sobre todo por el empuje de un sector tan masculinizado como el de la construcción. Sin embargo,

22 IGLESIAS, Juan et alii. La población de origen ecuatoriano en España. Características, necesidades y expectativas en tiempo de crisis.

${ }^{23}$ LÓPEZ de LERA, Diego. El retorno migratorio en época de crisis. La situación española.

${ }^{24}$ GÓMEZ, Valeriano. Crisis, inmigración y política de empleo. Una visión de conjunto.

${ }^{25}$ LASHERAS, Rubén, ZUGASTI, Nerea. La crisis y el proceso migratorio; OLIVER, Josep. El mercado de trabajo de la inmigración 2007-2010. Los cambios 2009/2010 en el marco de la crisis.

${ }^{26}$ OLIVER, Josep. La mejoría del empleo inmigrante en España en 2014: ¿Cambios estructurales?, p. 127.

${ }^{27}$ GARCÍA PÉREZ, Ignacio, TRONCOSO, David. La inmigración en el mercado laboral español. ¿Qué ha cambiado entre 2007 y 2009?; AYSA-LASTRA, María, CACHÓN, Lorenzo. Latino Inmigrant Employment during the Great Recession: A Comparison of the United States and Spain.

${ }^{28}$ PAPADEMETRIOU, Demetrios G., TERRAZAS, Aaron. Immigrant and the Current Economic Crisis: Research Evidence, Policy Challenges and Implications.

${ }^{29}$ COLECTIVO IOÉ. Impacto de la crisis sobre la población inmigrante; GODENAU, Dirk et alii. La integración de los inmigrantes en España: una propuesta de medición a escala regional; FOESSA. VII. Informe sobre exclusión y desarrollo social en España. 
tras la crisis y el dramático descenso de la actividad en este sector, la tasa de ocupación del colectivo masculino inmigrante desciende en mayor medida que la de las mujeres, que se mantiene en la primera fase de la crisis -2008$2011-{ }^{30}$. Aunque en el segundo periodo las mujeres inmigrantes han sufrido el impacto del desempleo con mayor intensidad. De igual forma, también se han dado situaciones como el aumento de la tasa de actividad de ciertos colectivos femeninos, como por ejemplo el magrebí, que en el periodo previo a la crisis mostraba unas tasas de actividad muy bajas y que durante la crisis han visto aumentar dicha tasa, sobre todo como consecuencia del descenso de la ocupación masculina de esta procedencia y la necesidad de buscar ingresos adicionales a los del hombre cabeza de familia ${ }^{31}$.

En todo momento, se observa una situación subalterna de las mujeres inmigrantes dentro del mercado laboral, en la que tanto en el periodo previo como en el de crisis ocupa los puestos de trabajo más precarios y por lo tanto es más proclive a situaciones de discriminación, debido a la interacción de una serie de factores como la clase, el género o la etnia, como bien han subrayado a nivel internacional autoras como Morokvasic ${ }^{32}$ o Sassen $^{33}$ o Sonia Parella para España con su término de la triple discriminación ${ }^{34}$.

La situación del País Vasco con respecto a la inmigración de origen extranjero no es ajena a esta realidad y el peso de las mujeres inmigrantes dentro del colectivo y del mercado laboral es muy relevante. De hecho, el modelo migratorio vasco y sus características son un claro ejemplo de la feminización de los flujos migratorios y de su inserción dentro del mercado laboral, sobre todo a través del sector de las tareas domésticas y los cuidados personales ${ }^{35}$. Así lo corrobora un estudio realizado por el Instituto Vasco de la Mujer ${ }^{36}$, donde casi la mitad de las personas inmigrantes asentadas en la CAPV en los últimos diez años son mujeres. Mujeres que muestran diferencias en cuanto a su percepción de integración y discriminación según áreas geográficas de origen, el tiempo que llevan residiendo en la CAPV, su situación administrativa y la edad, entre otros. El empleo se erige como una de las principales motivaciones para iniciar el proyecto migratorio, y su llegada a la CAPV está marcada por varios factores, entre los que se encuentra la posibilidad de insertarse en ciertos nichos laborales, altamente feminizados, caracterizados por la preferencia de mujeres latinoamericanas.

${ }^{30}$ MUÑOZ, Jacobo. Evolución del empleo y del paro de las mujeres inmigrantes en el mercado de trabajo español. El impacto de la actual crisis económica.

31 COLECTIVO IOÉ. Crisis e inmigración marroquí en España. 2007-2011.

32 MOROKVASIC, Mirjana. Birds of Passage are also women.

${ }^{33}$ SASSEN, Saskia. Globalization and its discontents. Essays on the New Mobility and People and Money.

34 PARELLA, El trasvase..., op. cit.; IDEM. Mujer, Inmigrante y Trabajadora: La triple discriminación.

${ }_{35}$ MORENO, Gorka. El impacto de la crisis sobre el colectivo inmigrante en el País Vasco.

${ }^{36}$ AIERDI, Xabier, OLEAGA, José A. Mujeres inmigrantes extranjeras en la CAE. 


\section{Datos y metodología}

Para analizar la actividad laboral de la población femenina de origen extranjero en la CAPV nos hemos valido de la EPIE, encuesta dirigida a recabar información referente a las condiciones de vida de la población extranjera de origen, tanto comunitario como de terceros países. Dicha encuesta está realizada en los años 2010 y 2014. Se posee, por tanto, información de dos momentos históricos distintos, lo que posibilita efectuar un análisis en el tiempo ante variables de carácter general, de su proyecto migratorio y motivos por los que lo llevó a cabo, de sus necesidades y de su nivel de integración en la CAPV.

La muestra en la EPIE 2010 es de 4.739 individuos de origen extranjero, que aplicándole la ponderación correspondiente serían 179.582 individuos, con un error muestral de $+-1,59 \%$. En el caso de la EPIE 2014 es de 4.873 individuos de origen extranjero, que aplicándole la ponderación serían 195.246, con un error muestral de en este caso $+-1,58 \%$.

Entre toda la información recabada en esta encuesta, nos detendremos a analizar aquellos datos relacionados con la actividad laboral, ocupación y paro. Para ello tomamos como referencia a la población de origen extranjero en edad potencialmente activa, esta es, de los 16 hasta los 66 años. Tendremos por tanto en el año 2010 a 147.790 personas de origen extranjero en esta franja de edad, donde 70.394 son hombres $(47,6 \%$ ) y 77.396 mujeres $(52,4 \%)$. En el año 2014 aumenta hasta las 155.685 personas, en el que 70.629 son hombres $(45,4 \%)$ y 85.057 son mujeres $(54,6 \%)$.

La técnica de análisis de datos es descriptiva para conocer la tasa de actividad, la tasa de paro y el coeficiente de ocupación, así como los principales sectores de actividad en donde se incorpora la población femenina de origen extranjero, prestando especial atención al servicio doméstico. Las variables utilizadas para este artículo son la situación de actividad, la ocupación u oficio actual, analizadas según sexo y área geográfica de origen.

\section{Resultados}

\subsection{Relación con la actividad, ocupación y paro}

En primer lugar vamos a analizar la situación del colectivo inmigrante en el mercado laboral del País Vasco y su relación con el empleo y actividad. Todo ello teniendo en cuenta el impacto que ha tenido sobre la mujer inmigrante y sus distintas procedencias entre los años de crisis económica (datos de 2010 y 2014). Para ello, en este apartado analizaremos los datos sobre la actividad, ocupación y paro de la población extranjera a partir de los resultados obtenidos en la EPIE. Por un lado disponemos de los datos referidos al año 2010, que se enmarca en los primeros años de la recesión económica -en el País Vasco dicha recesión se da de forma algo más tardía que en el resto de España, 
principalmente como consecuencia de una menor relevancia del sector de la construcción-; y el año 2014, que coincide con el segundo periodo de la crisis económica y con el momento de mayor intensidad de esta en el País Vasco.

Tabla 1. Población de 16 a 66 años. Indicadores generales de actividad, ocupación y paro de la población de origen extranjero, por área de origen y sexo, $2014^{37}$

\begin{tabular}{|c|c|c|c|c|c|c|c|c|c|}
\hline \multicolumn{10}{|c|}{2014} \\
\hline \multirow[t]{2}{*}{ Área de origen } & \multicolumn{3}{|c|}{ TASA DE ACTIVIDAD } & \multicolumn{3}{|c|}{$\begin{array}{c}\text { COEFICIENTE DE } \\
\text { OCUPACIÓN }\end{array}$} & \multicolumn{3}{|c|}{ TASA DE PARO } \\
\hline & Hombre & Mujer & Total & Hombre & Mujer & Total & Hombre & Mujer & Total \\
\hline UE Occidental & 81,2 & 65,6 & 73,8 & 64,7 & 53,3 & 59,3 & 20,3 & 18,7 & 19,6 \\
\hline $\begin{array}{l}\text { Rumanía y otros UE } \\
\text { Oriental }\end{array}$ & 89,7 & 75,5 & 81,5 & 57,5 & 48,6 & 52,4 & 35,9 & 35,7 & 35,8 \\
\hline Magreb & 84,6 & 58,8 & 74,8 & 26,5 & 19,0 & 23,6 & 68,7 & 67,7 & 68,4 \\
\hline $\begin{array}{l}\text { Argentina, Chile, } \\
\text { Uruguay }\end{array}$ & 87,6 & 70,4 & 78,2 & 73,6 & 48,3 & 59,9 & 16,0 & 31,3 & 23,5 \\
\hline $\begin{array}{l}\text { Colombia, Ecuador, } \\
\text { Perú }\end{array}$ & 80,4 & 76,6 & 78,0 & 50,7 & 52,7 & 51,9 & 37,0 & 31,1 & 33,4 \\
\hline Bolivia & 86,5 & 87,7 & 87,3 & 44,2 & 62,6 & 56,3 & 48,9 & 28,6 & 35,5 \\
\hline Paraguay & 84,0 & 92,9 & 90,6 & 55,9 & 80,9 & 74,5 & 33,5 & 12,9 & 17,8 \\
\hline $\begin{array}{l}\text { Brasil, Venezuela, } \\
\text { R.Dominicana }\end{array}$ & 85,8 & 74,4 & 78,0 & 54,4 & 49,4 & 51,0 & 36,5 & 33,6 & 34,6 \\
\hline Resto América Latina & 86,9 & 84,6 & 85,2 & 52,6 & 64,4 & 61,0 & 39,4 & 23,9 & 28,4 \\
\hline China & 88,5 & 82,2 & 85,2 & 86,1 & 80,0 & 82,9 & 2,7 & 2,7 & 2,7 \\
\hline Senegal & 87,5 & 69,5 & 84,3 & 34,2 & 19,7 & 31,6 & 60,9 & 71,7 & 62,5 \\
\hline Resto de África & 91,3 & 76,3 & 85,5 & 20,1 & 9,5 & 16,0 & 78,0 & 87,5 & 81,2 \\
\hline Resto del Mundo & 87,6 & 68,0 & 77,6 & 43,2 & 44,6 & 43,9 & 50,7 & 34,5 & 43,4 \\
\hline Total & 85,5 & 75,0 & 79,8 & 46,8 & 49,7 & 48,4 & 45,3 & 33,8 & 39,4 \\
\hline
\end{tabular}

Fuente: Elaboración propia a partir de la EPIE (2014). Gobierno Vasco.

La participación de las personas inmigrantes en el mercado laboral influye en gran medida en el proceso de vital y de integración social. La tabla 1 muestra, en primer lugar, como en 2014 la mayor parte de la población de origen extranjero $(79,8 \%)$ se encuentra en disposición de trabajar. Este es un dato recurrente dentro del colectivo inmigrante, que muestra unas tasas de actividad sensiblemente mayores a la población autóctona, tanto a nivel estatal como regional y tanto antes como durante el periodo de recesión. Como puede pensarse, la variable demográfica y las motivaciones migratorias influyen en estas

\footnotetext{
${ }^{37}$ La tasa de actividad es el cociente entre el número total de la población activa (paradas y ocupadas) y la población total; la tasa de paro es el cociente entre el número de personas paradas y el de personas activas (paradas y ocupadas); el coeficiente de ocupación es la proporción entre la población ocupada en la población total de referencia (INE).
} 
diferencias. Por sexo, los hombres de origen extranjero muestran unas tasas de actividad superiores $(85,5 \%)$ a las de las mujeres $(75 \%)$, aunque con importantes diferencias según la procedencia, tal y como se analizará en el próximo párrafo.

Por principales procedencias podemos constatar cómo apenas hay diferencias entre los hombres, con tasas de actividad similares. Sin embargo, entre las mujeres inmigrantes podemos advertir diferencias significativas. Así, por una parte, encontramos las procedencias de origen latinoamericano, con altas tasas de actividad, que están por encima de la media, destacando el caso de las paraguayas $(92,9 \%)$, bolivianas $(87,7 \%)$, y también las chinas $(82,2 \%)$. Mientras que, en el lado opuesto, nos encontramos con las mujeres procedentes del Magreb, con unas bajas tasas de actividad (58,8\%).

En segundo lugar, aproximadamente la mitad de las personas que están en disposición de trabajar se encuentran ocupadas $(48,4 \%)$. Aunque a primera vista, no hay grandes diferencias entre hombres y mujeres, la ocupación es algo mayor entre las mujeres $(49,7 \%)$ que entre los hombres (46,8\%). Este es un dato relevante, ya que como hemos visto, las mujeres muestran una tasa de actividad diez puntos porcentuales por debajo que los hombres. Sin embargo, cuando analizamos la ocupación vemos que estos porcentajes se equiparan e incluso son favorables a las mujeres. Todo ello apunta a una mayor empleabilidad e inserción laboral de las mujeres inmigrantes para el caso del País Vasco.

Cuando ponemos el acento en la variable origen de procedencia, otra vez, vemos cómo hay una relación entre origen y acceso al mercado laboral. De hecho, la población de origen africano tiene dificultades para acceder al mercado laboral mayor que el resto de las procedencias. Estas dificultades se acentúan entre las mujeres con unas tasas de ocupación menores que las de los hombres, como ocurre entre las mujeres magrebíes con tasas de ocupación del $19 \%$ frente el $26,5 \%$ de los hombres de su mismo origen.

Esta situación contrasta con la de la población de origen latinoamericano con tasas de ocupación no sólo superiores a la media, sino también más altas que las que tienen los hombres de sus mismas áreas de origen. De hecho, en la inmensa mayoría del resto de procedencias el hombre tiene una tasa de ocupación mayor que las mujeres. Sin embargo, dentro del origen latinoamericano esta tendencia se invierte y en algunos casos se dan diferencias muy considerables, como es el caso de Paraguay, en el que las mujeres tienen una tasa de ocupación del 80,9\% frente al 55,9\% de los hombres; o el caso de Bolivia, con un $62,6 \%$ para las mujeres y un $44,2 \%$ para los hombres.

Para terminar, podemos decir que la población china roza prácticamente el pleno empleo, con tasas de ocupación algo superiores entre los hombres $(86,1 \%)$ que entre las mujeres $(80 \%)$, mostrando así una pauta diferencia muy marcada con la mayoría del resto del colectivo inmigrante y en muchos casos 
situándose muy cerca de parámetros de población autóctona en variables de corte laboral y socioeconómico.

Lógicamente, los datos de paro están estrechamente vinculados a los ya mencionados de la actividad y la ocupación. De esta forma, una de cada cuatro personas extranjeras en disposición de trabajar está desempleada (39,4\%), siendo mayor entre los hombres (45,3\%) que entre las mujeres (33,8\%). Esta pauta, aparece muy marcada, sobre todo, entre la mayoría de las procedencias latinoamericanas. En el caso de la población paraguaya el desempleo de las mujeres se sitúa en el $12,9 \%$, frente al $33,5 \%$ de los hombres; y en el de las personas de origen boliviano, un $28,6 \%$ para las mujeres y un $48,9 \%$ para los hombres.

La situación de otros colectivo femenino es bien diferente, sobre todo en el caso de la población de origen africano, en la que junto a unas tasas de actividad menores aparecen unas de desempleo muy altas. En el caso de la mujer magrebí el paro llega a un $67,7 \%$ y en el de la senegalesa y resto de África a un $71,7 \%$ y un $87,5 \%$, respectivamente.

De esta forma, puede observarse como la empleabilidad de las mujeres inmigrantes en el País Vasco es muy desigual y como pueden observarse dos grandes polos. Las mujeres latinoamericanas muestran unas tasas de actividad y ocupación altas, unidas a un desempleo bajo. Por el contrario, las mujeres africanas son las que muestran una peor inserción laboral, con unas tasas de actividad y ocupación bajas y una tasa de desempleo muy alta.

\section{Gráfico 1. Población de 16 a 66 años. Tasa de actividad, coeficiente de ocupación y tasa de paro de la población de origen extranjero en la CAPV (2010 y 2014) (\%)}

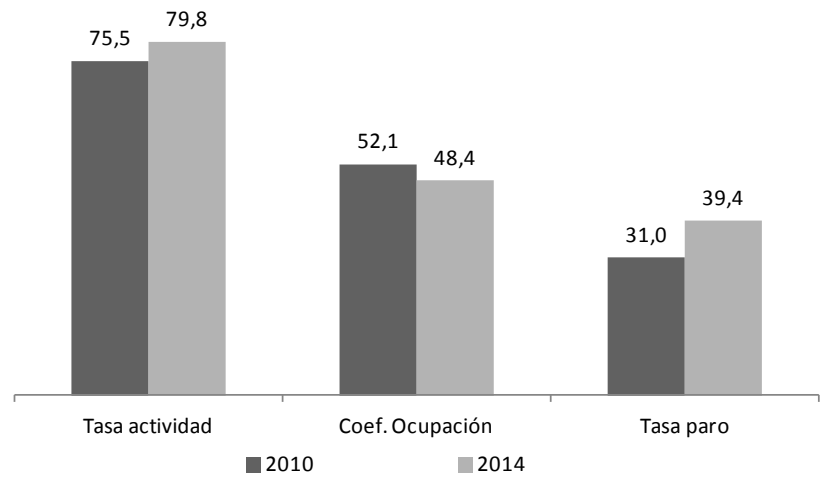

Fuente: Elaboración propia a partir de la EPIE (2010, 2014). Gobierno Vasco.

Pasando ya a analizar el impacto de la crisis en los periodos 2010 y 2014 (gráfico 1) y los efectos de ésta sobre el acceso al mercado laboral. En primer lugar, puede subrayarse un ligero aumento de la tasas de actividad (+4,3 puntos), al tiempo que ha descendido el coeficiente de ocupación (-3,7 puntos) y aumentado 
la tasa de paro ( $+8,4$ puntos). Esta pauta no sólo se da para el País Vasco, sino que se ha podido observar en todo el conjunto español, de tal forma que la crisis hace que población inactiva pase a activa, como respuesta a la situación socioeconómica y a la necesidad de buscar estrategias familiares de respuesta al impacto del aumento del desempleo entre la población anteriormente ocupada.

Gráfico 2. Tasa de actividad, ocupación y paro de las mujeres inmigrantes en el País Vasco (16 a 66 años) según principales procedencias (2010-2014) (\%)

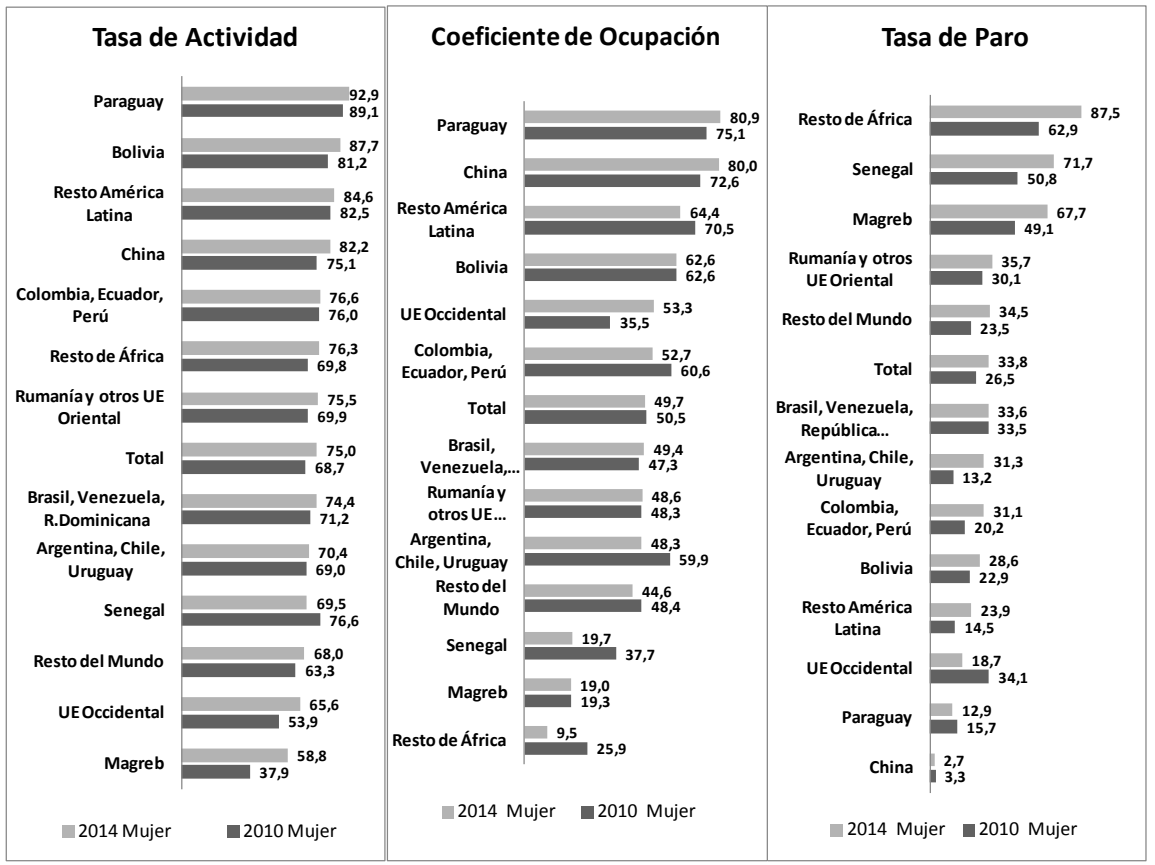

Fuente: Elaboración propia a partir de la EPIE (2010, 2014). Gobierno Vasco.

Sin embargo, y como se observa en la tabla 2, la evolución en el acceso al mercado laboral ha sido distinto en función del sexo y de las distintas procedencias. La tasa de actividad de las mujeres inmigrantes ha aumentado en mayor medida ( $+6,3$ puntos) que la de los hombres $(+2,6$ puntos). Destacando, sobre todo, el caso de las mujeres magrebíes, ya que estas han pasado de tener una baja tasa de actividad en 2010 con un $37,9 \%$ a un 58,8\% en 2014 (gráfico 2). Este es un dato interesante de analizar, ya que la crisis ha supuesto para las mujeres magrebíes un acceso al mercado laboral que, seguramente en otras circunstancias no se hubiera dado, debido sobre todo a pautas culturales y sociales que prevalecen dentro del colectivo. Como puede pensarse, en estos dos años (2010-2014) se ha dado un ligero descenso del coeficiente de ocupación de la población de origen extranjero. Este descenso 
se ha dado sobre todo entre los hombres, más en los de procedencia rumana (-9,3 ptos.) y magrebí (-13,2 ptos.), muy influido por el impacto del desempleo en el sector de la construcción; y menos entre las mujeres, en general. Así, puede observarse cómo la crisis en el País Vasco ha afectado sobre todo a los hombres, pasando su tasa de ocupación de un 53,9\% en 2010 a un 46,8\% en 2014; mientras que dichos porcentajes en el caso de las mujeres se mantienen muy estables, un 50,5\% para 2010 y un $49,7 \%$ para 2014. Dicho de otra forma, el impacto de la crisis en el mercado laboral sobre las mujeres inmigrantes en el País Vasco ha sido muy leve y han mantenido una ocupación muy similar a los inicios del periodo de recesión económica.

Tabla 2. Población de 16 a 66 años. Diferencias en tasa de actividad, ocupación y paro entre hombres y mujeres inmigrantes en el País Vasco según principales procedencias (2010-2014) (\%)

\begin{tabular}{|c|c|c|c|c|c|c|c|c|}
\hline \multicolumn{3}{|c|}{ Diferencias tasa de actividad } & \multicolumn{3}{|c|}{ Diferencias c. de ocupación } & \multicolumn{3}{|c|}{ Diferencias tasa de paro } \\
\hline Área de origen & $\begin{array}{c}\text { Difa } \\
\text { Hombre } \\
2010- \\
2014\end{array}$ & $\begin{array}{l}\text { Difa } \\
\text { Mujer } \\
2010- \\
2014\end{array}$ & Areas de orígen & $\begin{array}{l}\text { Difa } \\
\text { Hombre } \\
2010- \\
2014\end{array}$ & $\begin{array}{l}\text { Difa } \\
\text { Mujer } \\
2010- \\
2014\end{array}$ & Areas de orígen & $\begin{array}{c}\text { Difa } \\
\text { Hombre } \\
2010- \\
2014\end{array}$ & $\begin{array}{l}\text { Difa } \\
\text { Mujer } \\
2010- \\
2014\end{array}$ \\
\hline Senegal & $-2,2$ & $-7,1$ & Senegal & $-9,1$ & $-18,0$ & UE Occidental & $-7,6$ & $-15,4$ \\
\hline $\begin{array}{l}\text { Colombia, } \\
\text { Ecuador, Perú }\end{array}$ & $-0,1$ & 0,6 & Resto de África & $-13,6$ & $-16,4$ & Paraguay & $-2,2$ & $-2,8$ \\
\hline $\begin{array}{c}\text { Argentina, } \\
\text { Chile, Uruguay }\end{array}$ & 4,3 & 1,4 & $\begin{array}{c}\text { Argentina, } \\
\text { Chile, Uruguay }\end{array}$ & 11,6 & $-11,6$ & China & $-4,0$ & $-0,6$ \\
\hline $\begin{array}{l}\text { Resto América } \\
\text { Latina }\end{array}$ & 5,7 & 2,1 & $\begin{array}{c}\text { Colombia, } \\
\text { Ecuador, Perú }\end{array}$ & $-6,8$ & $-7,9$ & $\begin{array}{c}\text { Brasil, } \\
\text { Venezuela, R. } \\
\text { Dominicana }\end{array}$ & 2,7 & 0,1 \\
\hline $\begin{array}{c}\text { Brasil, } \\
\text { Venezuela, R. } \\
\text { Dominic. }\end{array}$ & 20,6 & 3,2 & $\begin{array}{l}\text { Resto América } \\
\text { Latina }\end{array}$ & 8,8 & $-6,1$ & $\begin{array}{c}\text { Rumanía y otros } \\
\text { UE Oriental }\end{array}$ & 9,5 & 5,6 \\
\hline Paraguay & 1,2 & 3,8 & $\begin{array}{l}\text { Resto del } \\
\text { Mundo }\end{array}$ & $-18,8$ & $-3,8$ & Bolivia & 15,5 & 5,7 \\
\hline $\begin{array}{l}\text { Resto del } \\
\text { Mundo }\end{array}$ & $-5,2$ & 4,7 & Total & $-7,1$ & $-0,8$ & Total & 10,3 & 7,3 \\
\hline $\begin{array}{l}\text { Rumanía y otros } \\
\text { UE Oriental }\end{array}$ & $-1,1$ & 5,6 & Magreb & $-13,2$ & $-0,3$ & $\begin{array}{l}\text { Resto América } \\
\text { Latina }\end{array}$ & $-6,6$ & 9,4 \\
\hline Total & 2,6 & 6,3 & Bolivia & $-4,7$ & 0,0 & $\begin{array}{c}\text { Colombia, } \\
\text { Ecuador, Perú }\end{array}$ & 8,4 & 10,9 \\
\hline Resto de África & 14,1 & 6,5 & $\begin{array}{c}\text { Rumanía y otros } \\
\text { UE Oriental }\end{array}$ & $-9,3$ & 0,3 & $\begin{array}{l}\text { Resto del } \\
\text { Mundo }\end{array}$ & 17,5 & 11,0 \\
\hline Bolivia & 13,1 & 6,5 & $\begin{array}{c}\text { Brasil, } \\
\text { Venezuela, R. } \\
\text { Dominicana }\end{array}$ & 11,2 & 2,1 & $\begin{array}{c}\text { Argentina, } \\
\text { Chile, Uruguay }\end{array}$ & $-9,6$ & 18,1 \\
\hline China & $-3,4$ & 7,1 & Paraguay & 2,7 & 5,8 & Magreb & 16,8 & 18,6 \\
\hline UE Occidental & $-4,6$ & 11,7 & China & 0,4 & 7,4 & Senegal & 9,2 & 20,9 \\
\hline Magreb & 2,0 & 20,9 & UE Occidental & 2,9 & 17,8 & Resto de África & 21,7 & 24,6 \\
\hline
\end{tabular}

Fuente: Elaboración propia a partir de la EPIE (2010, 2014). Gobierno Vasco. 
También es resaltable que las mujeres magrebíes tienen ahora mayores tasas de actividad, pero su coeficiente de ocupación continúa siendo bajo (19\%). Dicho de otra manera, y siguiendo con la lógica del párrafo anterior, han accedido al mercado laboral, pero muestran, al igual que antes, grandes dificultades para acceder a un empleo. Finalmente, la tasa de paro ha crecido y es mayor entre los hombres que entre las mujeres inmigrantes (gráfico 3). Sobre todo, la incidencia del paro ha sido mayor entre la población de origen magrebí, tanto entre los hombres como entre las mujeres. Así como también entre los hombres de procedencia rumana (9,5 puntos), boliviana $(15,5$ puntos), colombiana ( 8,4 puntos), y senegalesa (8,2 puntos). En definitiva, entre las mujeres inmigrantes, las tasas de paro más altas y las que más se han incrementado entre 2010 y 2014 corresponden a las mujeres magrebíes y senegalesas, mientras que las mujeres procedentes de Latinoamérica continúan teniendo unas tasas de paro por debajo de la media.

\subsection{Sectores de actividad: hombres, mujeres y tareas domésticas}

Los sectores de actividad donde trabajan la mayor parte de las mujeres inmigrantes son tres: tareas domésticas $(50,2 \%)$, hostelería $(14,9 \%)$ o comercio $(11,3 \%)$. El alto porcentaje de mujeres que trabaja en el sector de las tareas domésticas y cuidados personales deja patente cómo este sector se erige en el País Vasco como uno de los principales nichos laborales para las mujeres inmigrantes (gráfico 3).

En el caso de los hombres hay una mayor dispersión. Así, tres de cada cuatro que están trabajando lo hacen en sectores como la construcción (28,3\%), el comercio $(18,9 \%)$, la hostelería $(11,5 \%)$, los servicios a empresas $(5,2 \%)$, el transporte y comunicaciones $(6,6 \%)$ y la metalurgia $(4,9 \%)$.

Como ya hemos visto en indicadores anteriores, la presencia de las mujeres inmigrantes en los sectores de actividad difiere dependiendo de su procedencia. En este sentido, en el sector de las tareas domésticas predomina de manera muy marcada la mujer proveniente de Latinoamérica, debido en gran parte a una mayor similitud en las pautas culturales de cuidado y relaciones sociales y también a la coincidencia idiomática, que facilita el trato y los cuidados. En este origen algunos datos son muy ilustrativos. Un 87,6\% de las mujeres paraguayas ocupadas y un $68,9 \%$ de las bolivianas trabaja en este sector.

En el resto de procedencias, el peso de este sector es sensiblemente inferior, un 39\% de las mujeres senegalesas trabajan en las tareas domésticas, un $34,5 \%$ de las europeas orientales y un $18,2 \%$ de las europeas occidentales. 
Gráfico 3. Sectores de actividad de población de origen extranjero ocupada (16 a 66 años), por sexo en la CAPV, 2010 -2014 y diferencia 2010-2014 (\%)

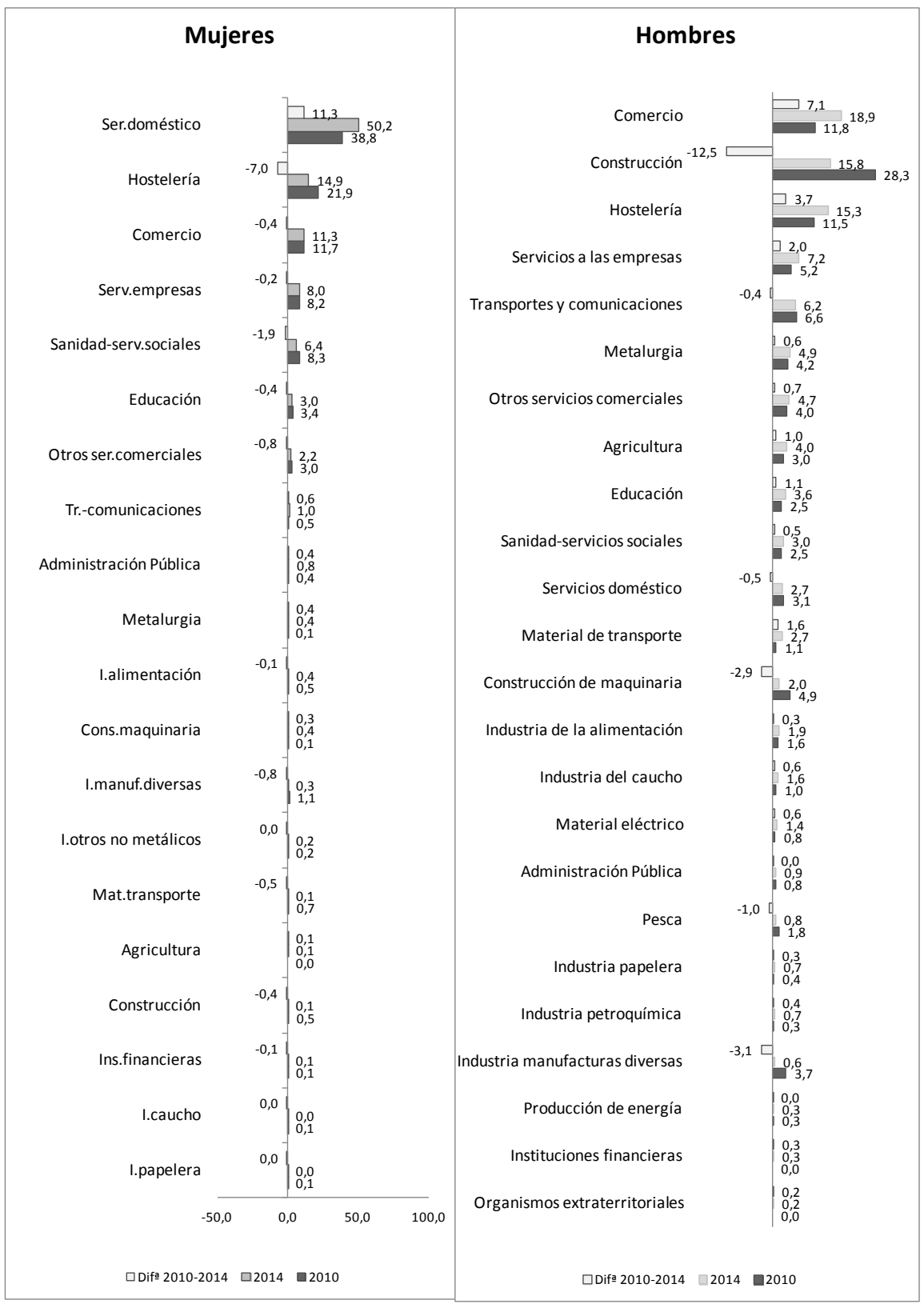

Fuente: Elaboración propia a partir de la EPIE (2010, 2014). Gobierno Vasco. 
En los años transcurridos de crisis económica en el País Vasco, entre 2010 y 2014 podemos constatar cómo el sector de las tareas domésticas se ha convertido en el nicho laboral refugio para una gran parte de las mujeres inmigrantes, descendiendo la presencia de estas en sectores como la hostelería o el comercio. Mientras que lo ocurrido entre los hombres inmigrantes ha sido, sobre todo, un desplome en el sector de la construcción -pasando del $28,3 \%$ al $15,8 \%$ - y en sectores relacionados como el sector de la maquinaria, aumentando su presencia en sectores como la hostelería, el comercio y servicios a empresas, entre otros.

Dentro del sector de las tareas domésticas también se detectan algunos cambios a lo largo del tiempo que son interesantes de subrayar (gráfico 4). En primer lugar es resaltable algo que ya se apunta en el párrafo anterior, el peso del servicio doméstico dentro de las mujeres inmigrantes ocupadas pasa de un $38,8 \%$ en 2010 a un $50,2 \%$ en 2014 . Este incremento se da en la mayoría de los principales orígenes y muestra cómo este sector se mantiene en el periodo de recesión y hace frente mejor a los envites que ésta ha producido dentro del mercado laboral, y más concretamente en el caso de las mujeres inmigrantes.

\section{Gráfico 4. Mujeres inmigrantes (16 a 66 años) ocupadas en el servicio doméstico según principales procedencias (2010-2014) (\%)}

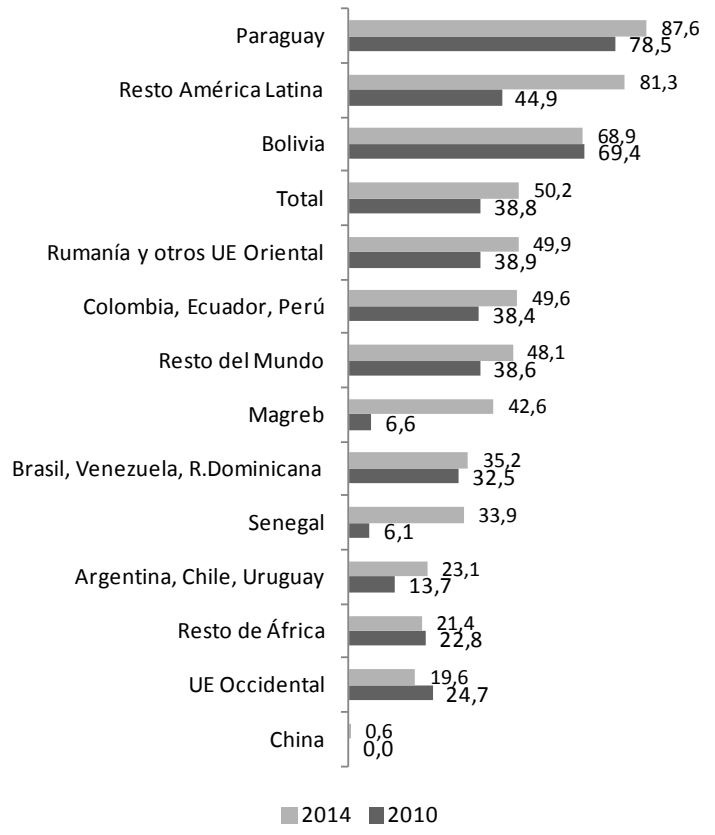

Fuente: Elaboración propia a partir de la EPIE (2010, 2014). Gobierno Vasco. 
En el caso de los orígenes en los que el peso de este sector era muy relevante sigue siéndolo e incluso aumenta su peso, como es el caso de las mujeres paraguayas, de las que un 78,5\% trabajaban en este sector en 2010 y eran un $87,6 \%$ en el 2014. Muestran una tendencia similar otras procedencias latinoamericanas o las mujeres de Europa oriental, que pasan de un 38,9\% a un $49,9 \%$ en 2014. Por último, resulta destacable también el aumento que se da en el caso de la población africana, que aunque presenten unas tasas de ocupación sensiblemente peores que el resto de procedencias, han pasado a trabajar con un mayor peso en el sector de las tareas domésticas. Así, un 6,6\% de las mujeres magrebíes ocupadas trabajan en este sector en el 2010, cuando en el 2014 este porcentaje ascendía hasta un 42,6\%. Algo similar se perfila también en el caso de las mujeres senegalesas y del resto de África.

\section{Conclusiones}

A través de este artículo hemos querido profundizar en el acceso al mercado laboral de las mujeres inmigrantes y hacer hincapié también en los efectos que el periodo de recesión ha producido en dicho acceso.

En primer lugar, es interesante destacar cómo en el País Vasco los hombres inmigrantes muestran unas tasas de actividad mayores que el de las mujeres. Sin embargo, este hecho no redunda a su vez en una mayor ocupación. De hecho, en el año 2014 las mujeres inmigrantes tenían una tasa de ocupación de un $49,7 \%$, frente al $46,8 \%$ de los hombres. Aunque la diferencia no es muy significativa, sí que es importante subrayar esta mayor empleabilidad de ellas frente a ellos. El origen de procedencia es una variable determinante también en este caso. De tal forma que son las mujeres latinoamericanas las que poseen una mayor ocupación, en la mayoría de los casos muy por encima de la que muestran los hombres de este mismo origen.

Los datos erigen un escenario en el que las mujeres latinoamericanas son el colectivo que mayor ocupación tiene en el mercado laboral vasco, a excepción del colectivo chino, que posee una ocupación incluso cercana a la de la población autóctona; aun y todo el peso de esta procedencia es sensiblemente menor al de Latinoamérica. Dicho de otra manera, entre todas las áreas geográficas de origen, son las mujeres latinoamericanas las que tienen más oportunidades de acceder al mercado laboral en el País Vasco.

En el lado opuesto se sitúan las mujeres magrebíes, también relevante en términos cuantitativos, con unas tasas de actividad y ocupación de las más bajas de entre los colectivos femeninos inmigrantes. Al respecto, no podemos dejar a un lado las diferencias existentes en aspectos tales como el propio proyecto migratorio y su inicio o diferentes pautas culturales y sociales que muestran estos dos grupos. En este sentido, cabe subrayar que la ocupación de las mujeres 
marroquíes se sitúa en un $19 \%$ y la tasa de desempleo en un $67,7 \%$, además de mostrar una tasa de actividad sensiblemente menor al resto de procedencias. Estos datos muestran grandes dificultades de acceso al mercado laboral por un lado; y también unas altas tasas de vulnerabilidad una vez accedido a éste.

En el periodo de recesión, que en el caso de este artículo se ve reflejado a través del análisis de datos de 2010 y 2014, no se da un cambio trascendental de este modelo. Sin embargo, sí que detectan algunos cambios importantes.

Durante el periodo de crisis la ocupación de las mujeres inmigrantes se ha mantenido muy estable, la que desciende más es la de los hombres, impulsada sobre todo por la destrucción de empleo en el sector de la construcción. Así, parece que las mujeres inmigrantes aguantan mejor los envites de la crisis sobre el mercado laboral.

En este escenario, el sector de las tareas domésticas y los cuidados personales ha sido un sector que ha hecho frente a la crisis mejor que otros. Si en 2010 un 38,8\% de las mujeres inmigrantes ocupadas trabajaban en este sector, en el 2014 este dato se sitúa en el 50,2\%, es decir, de cada dos mujeres inmigrantes ocupadas en el País Vasco una trabaja en el sector de las tareas domésticas. Este hecho, ha supuesto, por ejemplo, un importante aumento de la ocupación de las mujeres magrebíes -y africanas en general- en este sector, que era prácticamente anecdótico para este colectivo en 2010. La crisis ha supuesto un importante aumento de la actividad de las mujeres magrebíes, que aunque no se ha visto secundada por un incremento similar en la ocupación, sí que ha hecho que el acceso al mercado laboral haya aumentado sensiblemente en este periodo, sobre todo para hacer frente al aumento del desempleo entre los hombres de este origen.

Este es un dato esperanzador ya que aunque las mujeres magrebíes muestran unas tasas de alta vulnerabilidad social y laboral, el aumento de la actividad en el periodo de crisis puede ser la antesala para una mayor ocupación en un momento de bonanza económica, siempre y cuando en un escenario de este tipo no haya una vuelta atrás y un descenso en la tasa de actividad.

En conclusión, la crisis parece que ha apuntalado aún más este modelo en el que las mujeres inmigrantes muestran más posibilidades de tener un empleo que los hombres. Y aunque es pronto para ser tajante, el escenario poscrisis que está emanando en los últimos tiempos, parece que no hace más que corroborar esta tesis, ya que en el caso del País Vasco se está detectando la llegada de más población latinoamericana -durante los años de recesión los flujos de esta procedencia se habían ralentizado-, sobre todo de nuevas procedencias, especialmente centroamericanas -Nicaragua y Honduras-. 


\section{Bibliografía}

AIERDI, Xabier; OLEAGA, José A. Mujeres inmigrantes extranjeras en la CAE. VitoriaGasteiz: Emakunde, 2012.

AYSA-LASTRA, María; CACHÓN, Lorenzo. Latino Inmigrant Employment during the Great Recession: A Comparison of the United States and Spain. Norteamerica, v. 7, n. 2, 2012, p. 7-42.

CACHÓN, Lorenzo. Integración de los inmigrantes a través del trabajo. Documentación Social, n. 132, 2004, p. 97-120.

CACHÓN, Lorenzo. Diez notas sobre la inmigración en España y una conclusión ni inesperado ni desesperada. Tribuna Americana, n. 4, 2004, p. 41-53.

CACHÓN, Lorenzo. La España inmigrante: marco discriminatorio, mercado de trabajo y políticas de integración. Barcelona: Anthropos, 2009.

CARRASCO, Concepción. Inmigración y mercado de trabajo. Informe 2011. Madrid: Ministerio de Asuntos Sociales, 2012.

CASTLES, Stephen; MILLER, Mark J. The Age of Migration. Hampshire: Palgrave MacMillan, 2003.

CEBOLLA, Héctor; GONZÁLEZ-FERRER, Amparo. La inmigración en España (20002007). De la gestión de los flujos a la integración de los inmigrantes. Madrid: Centro de Estudios Políticos y Constitucionales, 2008.

COLECTIVO IOÉ. Mujeres inmigrantes en España. Proyectos migratorios y trayectorias de género. Madrid: Oficina Regional para la Inmigración de la Comunidad de Madrid, 1998.

COLECTIVO IOÉ. Mujer, inmigración y trabajo. Madrid: Ministerio de Trabajo y Asuntos Sociales, 2001.

COLECTIVO IOÉ. Impacto de la crisis sobre la población inmigrante. Madrid: Organización Internacional para las Migraciones, 2012.

COLECTIVO IOÉ. Crisis e inmigración marroquí en España. 2007-2011. Madrid. 2012. Disponible en: <http://www.colectivoioe.org/uploads/16ed2b9a5f0868d c55be62fa17d667ca48a97980.pdf>. Acceso el 21.12.2016.

DIAZ GORFINKIEL, Magdalena El mercado de trabajo de los cuidados y la creación de las cadenas globales de cuidado: ¿cómo concilian las cuidadoras? Cuadernos de Relaciones Laborales, v. 26, n. 2, 2008, p. 71-89.

FOESSA. VII. Informe sobre exclusión y desarrollo social en España. Madrid: Fundación Foessa, 2014.

GARCÍA PÉREZ, Ignacio; TRONCOSO, David. La inmigración en el mercado laboral español. ¿Qué ha cambiado entre 2007 y 2009? In AJA, Eliseo; ARANGO, Joaquín; OLIVER, Josep (dirs.). Inmigración y crisis económica. Impactos actuales y perspectivas de futuro. Anuario de la inmigración en España. 2010. Barcelona: CIDOB, 2011.

GARCÍA, Cristina; SANTOS, Lourdes; VALENCIA, Nelcy. La construcción social del mercado laboral doméstico en España a comienzos del siglo XXI. Cuadernos de Relaciones Laborales, v. 32, n. 1, 2014, p. 101-131. 
GIL, Sandra; GONZALEZ-FERNÁNDEZ, Tania. International migration, public policies and domestic work: Latin American migrant women in the Spanish domestic work system. Women's Studies International Forum, n. 46, 2014, p. 13-23.

GLENN, Evelyn N. From Servitude to Service Work: The Historical Continuities of Woemen's Paid and Unpaid Reproductive Works. Signs, v. 18, n. 1, 1992, p. 1-44.

GODENAU, Dirk; RINKEN, Sebastian; MARTÍNEZ DE LIZARRONDO, Antidio; MORENO, Gorka. La integración de los inmigrantes en España: una propuesta de medición a escala regional. Madrid: Ministerio de Empleo y Seguridad Social, 2014.

GÓMEZ, Valeriano. Crisis, inmigración y política de empleo. Una visión de conjunto. In AJA, Eliseo; OLIVER, Josep (dirs.). Inmigración y crisis económica. Impactos actuales y perspectivas de futuro. Anuario de la inmigración en España 2010. Barcelona: CIDOB, 2011.

GREGORIO, Carmen. La migración femenina y su impacto en las relaciones de género. Madrid: Narcea, 1998.

HOSCHSCHILD, Arlie R. Las cadenas mundiales de afecto y asistencia y la plusvalía emocional. In GIDDENS, Anthony; HUTTON, Will (coords.). En el límite: la vida en el capitalismo global. Madrid: Tusquets, 2001, p. 187-208.

IGLESIAS, Juan; MORENO, Gorka; FERNÁNDEZ, Mercedes; OLEAGA, José Antonio. La población de origen ecuatoriano en España. Características, necesidades y expectativas en tiempo de crisis. Madrid: Embajada de Ecuador en España, 2015.

IZQUIERDO, Antonio. La inmigración inesperada: La población extranjera en España (1991-1995). Madrid: Trotta, 1995.

KOFMAN, Eleonore. Gendered Global Migrations. International Feminist Journal of Politics, v. 6, n. 4, 2004, p. 643-665.

LASHERAS, Rubén; ZUGASTI, Nerea. La crisis y el proceso migratorio. In LAPARRA, Miguel; PÉREZ ERANSUS, Begoña (coords.). El primer impacto de la crisis en la cohesión social de España. Madrid: Fundación Foessa, 2010.

LÓPEZ DE LERA, Diego. El retorno migratorio en época de crisis. La situación española. Seminario Internacional: La migración de retorno en América Latina: Nuevos desafíos para la política migratoria, 2-3 de octubre, Quito, 2012.

MARTÍNEZ BUJÁN, Raquel. iEl trabajo doméstico cuenta! Características y transformaciones del servicio doméstico en España. Migraciones. Publicación del Instituto Universitario de Estudios sobre Migraciones, n. 36, 2014, p. 275-305.

MOLPECERES, Laura. Situación laboral de las mujeres inmigrantes en España. Cuadernos de Relaciones Laborales, v. 30, n. 1, 2012, p. 91-113.

MORENO, Gorka. El impacto de la crisis sobre el colectivo inmigrante en el País Vasco. In MORENO, Gorka (ed.). Inmigración e impacto de la crisis. Anuario de la inmigración en el País Vasco 2013. Bilbao: Universidad del País Vasco, 2014.

MORRISON, Andrew R.; SCHIFF, Maurice; SJÖBLOM, Mirja. The international migration of women. Washington D.C.: World Bank, 2007. 
MUÑOZ, Jacobo. Evolución del empleo y del paro de las mujeres inmigrantes en el mercado de trabajo español. El impacto de la actual crisis económica. Cuadernos de Relaciones Laborales, v. 30, n. 1, 2012, p. 115-137.

OECD. International Migration Outlook. SOPEMI. Washington D. C.: Organization for Economic Co-operation and Development, 2007.

OIM. Estudio sobre la situación laboral de la mujer inmigrante en España. Madrid: Organización Internacional para las Migraciones, 2015.

OLIVER, Josep. El mercado de trabajo de la inmigración 2007-2010. Los cambios 2009/2010 en el marco de la crisis. In AJA, Eliseo; ARANGO, Joaquín; OLIVER, Josep (dirs.). Inmigración y crisis económica. Impactos actuales y perspectivas de futuro. Anuario de la inmigración en España. 2001. Barcelona: CIDOB, 2011.

OLIVER, Josep. La mejoría del empleo inmigrante en España en 2014: ¿Cambios estructurales?. In ARANGO, Joaquín; MOYA, David; OLIVER, Josep; SÁNCHEZMONTIJANO, Elena (dirs.). Flujos cambiantes, atonía institucional. Anuario de la inmigración en España. 2014. Barcelona: CIDOB, 2015.

MOROKVASIC, Mirjana. Birds of Passage are also women. International Migration Review, v. 18, n. 4, 1984, p. 886-907.

UNITED NATIONS. International Migration Report 2013. New York: United Nations, 2013.

OROZCO, Amaia. Cadenas Globales de Cuidados. Santo Domingo: UN-INSTRAW, 2007.

OSO, Laura. La migración hacia España de mujeres jefes del hogar. Madrid: Instituto de la Mujer, 1998.

PAJARES, Miguel. Inmigración y mercado de trabajo. Informe 2010. Madrid: Ministerio de Trabajo e Inmigración, 2010.

PAPADEMETRIOU, Demetrios G.; TERRAZAS, Aaron. Immigrant and the Current Economic Crisis: Research Evidence, Policy Challenges and Implications. Washington D.C.: Migration Policy Institute, 2009.

PARELLA, Sonia. El trasvase de desigualdades de clase y etnia entre mujeres: los servicios de proximidad. Papers, n. 60, 2000, p. 275-289.

PARELLA, Sonia. Mujer, Inmigrante y Trabajadora: La triple discriminación. Barcelona: Anthropos, 2004.

PARELLA, Sonia. Las lógicas de la discriminación de las mujeres inmigrantes en el mercado de trabajo. Mecanismos y repercusiones. Puntos de Vista, n. 5, 2006, p. 29-46.

PIORE, Michael. Birds of passage: migrant labor and industrial societies. Cambridge: Cambridge University Press, 1979.

PIPER, Nicola. Gender and Migration. Geneva: Global Commission on International Migration, 2005.

SASSEN, Saskia. Why Migration? Tesis contra los modelos de explicación al uso. In VV.AA. Extranjeros en el paraíso. Barcelona: Virus, 1994. 
SASSEN, Saskia. Globalization and its discontents. Essays on the New Mobility and People and Money. New York: New Press, 1998.

TORNS, Teresa. Los servicios de proximidad zun yacimiento de empleo? Revista de Treball Social, n. 147, 1997, p. 40-47.

ZLOTNIK, Hania. The Global Dimensions of Female Migration. Washington D.C.: Migration Policy Institute, 2003.

Recibido para publicación en 27.01.2017

Aceptado para publicación en 09.03.2017

Received for publication in January $27^{\text {th }}, 2017$

Accepted for publication in March 09 ${ }^{\text {th }}, 2017$

ISSN impresso 1980-8585

ISSN eletrônico 2237-9843

http://dx.doi.org/10.1590/1980-85852503880004909 